\title{
Plasmacytoma of the Clivus Presenting as Bilateral Sixth Nerve Palsy
}

\author{
1 Section of Otology and Laryngology, Department of Otolaryngology, \\ Massachusetts Eye and Ear Infirmary, Harvard Medical School, \\ Boston, Massachusetts, United States \\ 2 Section of Pathology, Department of Otolaryngology, Massachusetts \\ General Hospital, Harvard Medical School, Boston, Massachusetts, \\ United States \\ ${ }^{3}$ Section of Pathology, Department of Pathology, Massachusetts \\ General Hospital, Harvard Medical School, Boston, Massachusetts, \\ United States
}

Neil Kalwani ${ }^{1, *}$ Aaron K. Remenschneider ${ }^{1, *}$ William Faquin ${ }^{2} \quad$ Judith Ferry $^{3} \quad$ Eric H. Holbrook ${ }^{1}$

J Neurol Surg Rep 2015;76:e156-e159.

\author{
Address for correspondence Aaron K. Remenschneider, MD, MPH, \\ Massachusetts Eye and Ear Infirmary, 243 Charles Street, Boston MA, \\ 02114, United States \\ (e-mail: Aaron_Remenschneider@meei.harvard.edu).
}

\begin{abstract}
Keywords

- plasmacytoma

- clivus

- skull base

- endoscopic sinus surgery

Background and Importance Plasmacytomas are monoclonal proliferations of plasma cells that may arise within soft tissue or bone. The skull base is a rare site for plasmacytomas to occur, and few cases have been reported in the literature. When present in the skull base, plasmacytomas may result in cranial neuropathies and often progress to multiple myeloma more rapidly than other intracranial or skeletal plasmacytomas.

Clinical Presentation A 69-year-old man presented with a primary complaint of diplopia and an examination consistent with bilateral abducens nerve palsy. No other deficits were noted. Magnetic resonance imaging of the skull base demonstrated a large T1 isointense moderately enhancing lesion centered within the clivus. Endoscopic biopsy of the mass revealed sheets and aggregates of mature monoclonal plasma cells. The patient's initial systemic work-up revealed that this was a solitary lesion, and he was treated with radiation therapy to the skull base with a durable local effect at 18-month follow-up. Unfortunately he progressed to multiple myeloma with peripheral osteolytic lesions but has been stabilized on chemotherapeutics.

Conclusion The clivus is an unusual site for intracranial plasmacytomas, and enhancing lesions must be differentiated from chordoma. Characteristic findings on histopathology include an immunoglobulin light-chain restricted clonal proliferation of plasma cells. Treatment is most commonly radiotherapy with surgery reserved for biopsy and palliation. Clinicians should be aware of the increased risk of progression to multiple myeloma in skull base plasmacytomas.
\end{abstract}

\section{Background and Importance}

Plasmacytomas are characterized by monoclonal proliferations of plasma cells. They may arise from osseous (intramedullary) or

$\overline{{ }^{*} \text { Authors }}$ contributed equally to this work. nonosseous (extramedullary) sites. ${ }^{1,2}$ Multiple myeloma, or plasma cell myeloma, is a plasma cell neoplasm with diffuse marrow involvement and/or multifocal disease and represents one end of the disease continuum. ${ }^{2,3}$ Intramedullary plasmacytomas progress to multiple myeloma more frequently (up to $67 \%)$ than extramedullary plasmacytomas $(\sim 15 \%){ }^{4}$ received

November 2, 2014

accepted

March 12, 2015

published online

June 26, 2015
DOI http://dx.doi.org/ 10.1055/s-0035-1554930. ISSN 2193-6366. (c) 2015 Georg Thieme Verlag KG Stuttgart · New York
License terms

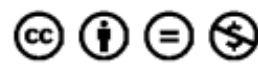




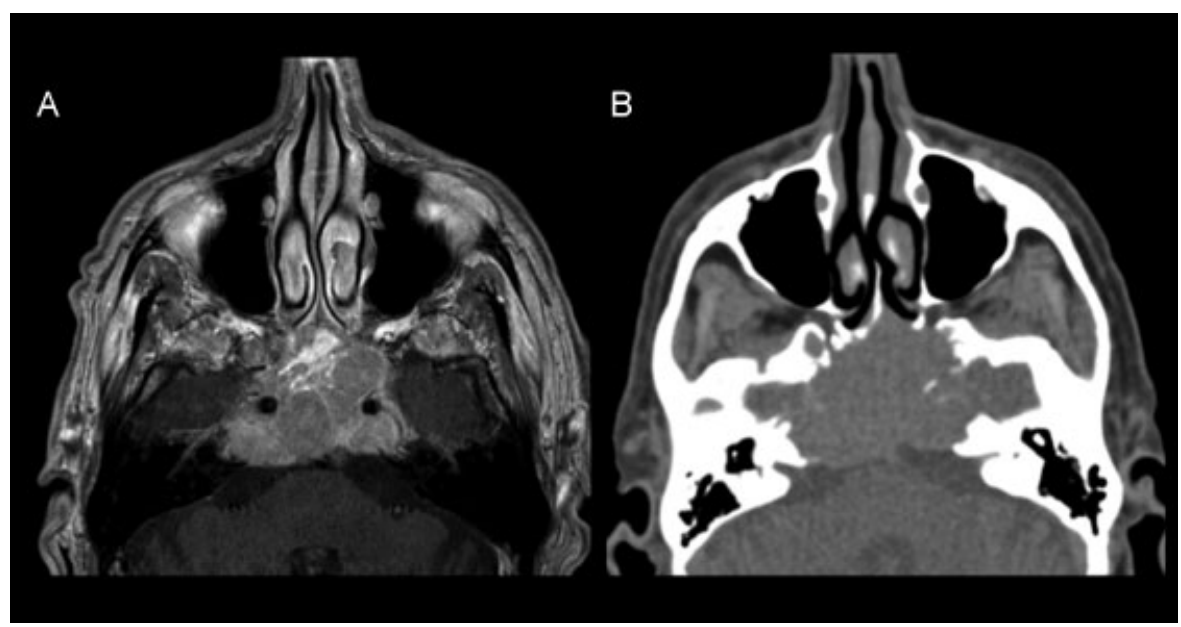

Fig. 1 (A) Preoperative magnetic resonance imaging showed a large T1 isointense moderately enhancing lesion centered within the clivus extending into the sphenoid, left posterior ethmoid cells, Meckel cave, and bilateral cavernous sinuses. (B) Computed tomography scan of the sinuses demonstrated an osteo-erosive mass centered within the clivus thinning the bone of the left lamina papyracea and optic canal.

The skull base is a rare site for plasmacytomas, and when it is involved, the most common sites are the orbit, sphenoid sinus, and dorsum sellae. ${ }^{5}$ Only 12 cases of clival plasmacytoma have been reported in the literature. ${ }^{5,6}$ Given that the clivus is rich in marrow, clival plasmacytomas are considered to be intramedullary. ${ }^{3}$ Plasmacytomas of the skull base progress to multiple myeloma more rapidly and have a poorer prognosis than other intracranial plasmacytomas. ${ }^{2,3,7} \mathrm{We}$ present a case of plasmacytoma of the clivus and then discuss the appropriate diagnosis and management of this lesion.

\section{Clinical Presentation}

A 69-year-old man presented with 1 week of diplopia. The physical examination revealed limitation of lateral extraocular movement in both eyes consistent with bilateral abducens nerve palsy. On endoscopic examination there was appreciable fullness within the superior nasopharynx with normal-appearing mucosa. The remainder of the examination was unremarkable.

Magnetic resonance imaging (MRI) of the skull base was obtained and showed a large T1 isointense moderately enhancing lesion $5.1 \times 3.5 \times 3.2 \mathrm{~cm}$ centered within the clivus extending into the sphenoid, left posterior ethmoid cells, Meckel cave, and bilateral cavernous sinuses (- Fig. 1A). A computed tomography (CT) scan of the sinuses demonstrated an osteo-erosive mass centered within the clivus thinning the bone of the left lamina papyracea and optic canal (-Fig. 1B).

The patient underwent an endoscopic left total ethmoidectomy. A salmon-colored mass was found extending from the area of the sphenoid into the posterior ethmoid air cells. Histologic examination showed sheets and aggregates of mature plasma cells underlying the epithelium (-Fig. 2). On immunohistochemical staining, the cells were found to express CD138 and CD56. They were diffusely positive for $\lambda$ light chain (-Fig. 3); к chain staining was negative (-Fig. 4).
Postoperatively, the patient's diplopia improved markedly. Initially, he was found to have a normal serum protein electrophoresis, no elevation of Bence-Jones protein in his urine, and a skeletal survey that did not show any new lytic or blastic lesions. He was treated with definitive radiation therapy at a dose of $30 \mathrm{~Gy}$ delivered in 2-Gy fractions, less than the ideal dose of 40 to $50 \mathrm{~Gy}$ given concerns regarding radiation toxicity. The patient's diplopia remains improved, and with the exception of new moderate symmetrical sensorineural hearing loss, he has not developed further neurologic or sinonasal symptoms in 18 months of follow-up.

A postradiation positron emission tomography (PET)-CT was performed that did not reveal increased avidity above background along the skull base; however, it did demonstrate several lytic lesions in the bone including his lumbar spine

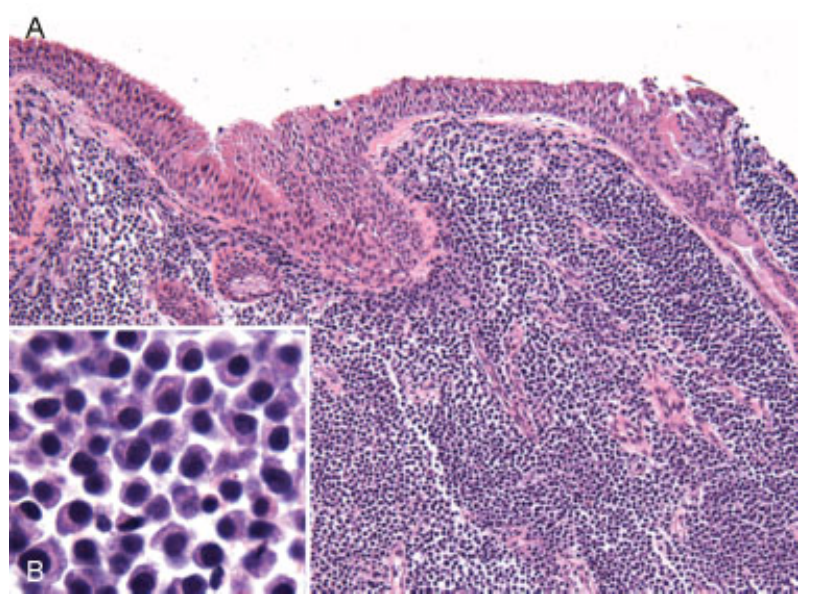

Fig. 2 (A) Low-power $(\times 100)$ hematoxylin and eosin stain revealed sheets and aggregates of mature plasma cells underlying the epithelium. (B) High-power $(\times 1,000)$ view demonstrated eccentric nuclei and clock face-like chromatin. 


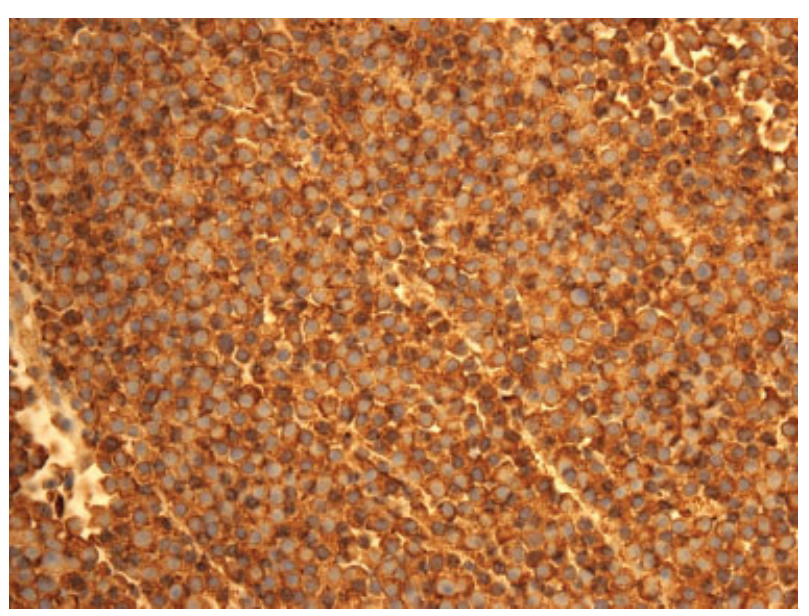

Fig. 3 Immunohistochemical staining revealed diffuse expression of $\lambda$ light chain.

and pelvis. He has progressed to develop oligo-secretory $\lambda$ light-chain multiple myeloma, International Staging System stage I. He experienced several pathologic extremity fractures and was treated with bortezomib and dexamethasone with radiographic stability of the peripheral lesions and improvement in pain.

\section{Discussion}

The mean age at symptom onset among the reported cases of clival plasmacytoma is 57 years with a female preponderance (female-to-male ratio: 2:1). The most common presenting symptoms are headache, visual deficits due to cranial nerve palsies, and endocrinologic abnormalities. ${ }^{5,6}$

On MRI, intracranial plasmacytomas enhance with gadolinium contrast and demonstrate an iso- to hyperintense signal on T1-weighted images and an iso- to hypointense signal on T2weighted images. ${ }^{1}$ On CT scan, the lesions are osseo-destructive and avidly enhance with intravenous contrast. Solitary clival

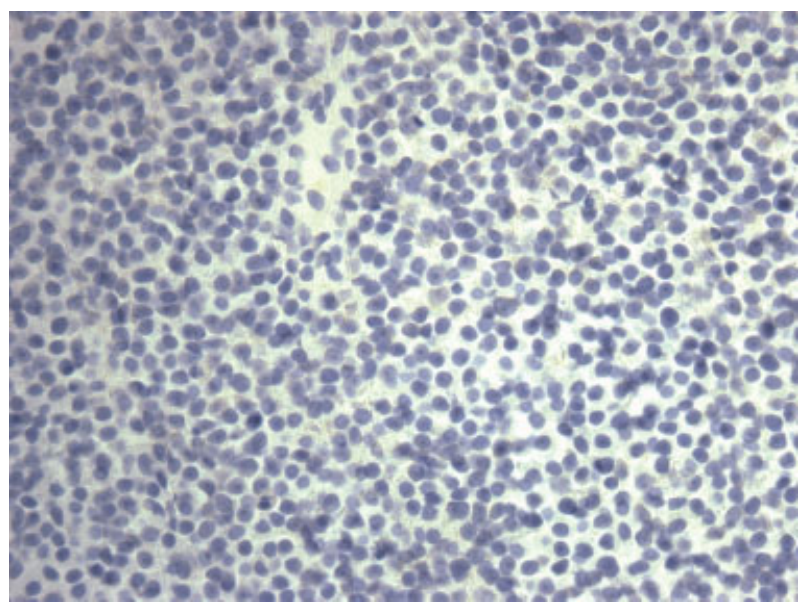

Fig. 4 Immunohistochemical staining did not show expression of $\kappa$ light chain. lesions are rare. The most frequent tumor at this site is chordoma (40\% of all cases). ${ }^{5}$ The radiologic differential should also include sarcoma, lymphoma, carcinoma, pituitary adenoma, meningioma, and metastatic disease. ${ }^{6}$

On microscopic examination, plasmacytomas consist of a proliferation of sheets of plasma cells with a few intervening cells of other types. Cytologic atypia is variable. The tumors may be composed predominantly of small mature plasma cells with small round nuclei with peripherally clumped ("clock-face") chromatin and inconspicuous nucleoli, or of larger cells with plasmablastic morphology, with large atypical nuclei with vesicular chromatin and prominent central nucleoli. Cytoplasm is typically moderately abundant and eccentrically oriented and may contain inclusions of condensed or crystallized immunoglobulin. A pale paranuclear Golgi region may be recognizable. Binucleated and multinucleated cells may be seen. Intranuclear protrusions of cytoplasm containing immunoglobulin called Dutcher bodies may occasionally be present. $^{6}$

Immunohistochemical staining shows expression of CD138 and CD38. ${ }^{5,6}$ Approximately $70 \%$ of the neoplastic plasma cells coexpress CD56 as well, a surface antigen that is not usually present on nonneoplastic plasma cells. A hallmark of plasmacytomas is the demonstration of immunoglobulin light-chain restriction, indicating that the proliferation is clonal. ${ }^{6,8,9}$

In addition to histologic evidence of a monoclonal plasma cell infiltrate, diagnosis of an isolated plasmacytoma requires demonstration of a lack of systemic involvement. ${ }^{10}$ This includes absent or low serum/urinary levels of monoclonal immunoglobulin, a normal skeletal survey, and a normal bone marrow biopsy and aspirate. ${ }^{2,3,5,7,10}$ Some authors also recommend a PET scan to rule out other sites of disease. Given the high rate of progression to multiple myeloma, patients with intramedullary plasmacytoma require careful followup. ${ }^{10}$ In the case of plasmacytoma of the skull base, conversion has been reported as late as 1 year after diagnosis. ${ }^{2}$

The recommended treatment for solitary clival plasmacytoma is radiation therapy given at a dose of 40 to 50 Gy over a 4-week period. ${ }^{1,2,6}$ Surgery serves primarily a diagnostic role because the benefit of extensive resection has not been determined. Surgical debulking is indicated when there is neurologic dysfunction secondary to the mass effect of the tumor. $^{6}$

Five-year disease-free and overall survival rates are 50\% and $74 \%$, respectively. Five-year local control following radiation therapy is relatively good at $86 \%$; treatment failure is more often due to systemic relapse. ${ }^{6}$ If multiple myeloma is diagnosed, chemotherapy or stem cell transplantation may be appropriate. ${ }^{11}$

\section{Conclusion}

The clivus is an unusual site for intracranial plasmacytomas. Radiation therapy alone is effective in achieving local control, with surgery serving primarily a diagnostic and palliative role. A full work-up for systemic disease and careful follow-up is required given the high risk of progression to multiple myeloma. 


\section{References}

1 Cerase A, Tarantino A, Gozzetti A, et al. Intracranial involvement in plasmacytomas and multiple myeloma: a pictorial essay. Neuroradiology 2008;50(8):665-674

2 Schwartz TH, Rhiew R, Isaacson SR, Orazi A, Bruce JN. Association between intracranial plasmacytoma and multiple myeloma: clinicopathological outcome study. Neurosurgery 2001;49(5): 1039-1044; discussion 1044-1045

3 Wein RO, Popat SR, Doerr TD, Dutcher PO. Plasma cell tumors of the skull base: four case reports and literature review. Skull Base 2002;12(2):77-86

4 McKenna R, Kyle R, Kuehl W, Grogan T, Harris N, Coupland R. Plasma cell neoplasms. In: Swerdlow S, Campo E, Harris N, et al, eds. WHO Classification Tumours of Haematopoietic and Lymphoid Tissues. 4th ed. Lyon, France: IARC; 2008:200-213

5 Gagliardi F, Boari N, Mortini P. Solitary nonchordomatous lesions of the clival bone: differential diagnosis and current therapeutic strategies. Neurosurg Rev 2013;36(4):513-522; discussion 522

6 Gagliardi F, Losa M, Boari N, et al. Solitary clival plasmocytomas: misleading clinical and radiological features of a rare pathology with a specific biological behaviour. Acta Neurochir (Wien) 2013; 155(10):1849-1856

7 Bindal AK, Bindal RK, van Loveren H, Sawaya R. Management of intracranial plasmacytoma. J Neurosurg 1995;83(2):218-221

8 Ustuner Z, Basaran M, Kiris T, et al. Skull base plasmacytoma in a patient with light chain myeloma. Skull Base 2003;13(3): 167-171

9 Yamaguchi S, Terasaka S, Ando S, Shinohara T, Iwasaki Y. Neoadjuvant therapy in a patient with clival plasmacytoma associated with multiple myeloma: a case report. Surg Neurol 2008;70(4): 403-407

10 Soutar R, Lucraft H, Jackson G, et al; Working Group of the UK Myeloma Forum; British Committee for Standards in Haematology; British Society for Haematology. Guidelines on the diagnosis and management of solitary plasmacytoma of bone and solitary extramedullary plasmacytoma. Clin Oncol (R Coll Radiol) 2004; 16(6):405-413

11 Nofsinger YC, Mirza N, Rowan PT, Lanza D, Weinstein G. Head and neck manifestations of plasma cell neoplasms. Laryngoscope 1997;107(6):741-746 